\title{
Main routes of the porous composite materials creation
}

\author{
P.G. Kudryavtsev iD \\ KUD Industries PN Ltd - Israel Nanotechnology Technology Research Center, Haifa, Israel \\ Corresponding author: e-mail: pgkudr89@gmail.com
}

\begin{abstract}
This paper is devoted to an overview of the main ways of creating porous composite materials. Porous materials are solids containing free space in the form of cavities, channels, or pores, which determine the presence of an internal interfacial surface. The analysis of the general methods of obtaining porous materials. A deposition is one of the most common methods for producing porous materials. Thermal decomposition, as a method used to obtain porous oxide materials by thermal decomposition of various compounds. Hydrothermal synthesis is widely used to produce zeolites. Selective dissolution of individual components of a substance using chemical reactions is also one of the effective methods for creating or increasing porosity. The paper discusses the methods of forming highly porous refractory materials. There are two main ways of forming refractory ceramic products. The first way is the direct sintering of dispersions of ceramic fibers. The second method is the use of a binder, which can significantly reduce the temperature of obtaining a porous product. The possibilities of obtaining porous nanocomposites based on aerogels are shown. Composite materials are usually obtained by combining two different materials. In general, the creation of composites is used to take advantage of each type of material and to minimize their disadvantages. Aerogels are fragile substances. But with the introduction of another component into their structure, it is possible to increase the strength of the material. Such materials have the desired optical properties, high surface area, and low density like silica aerogel. A review of methods for obtaining porous materials using the phenomenon of spinodal decomposition has been carried out. Materials whose structure is formed in microphase separation during polymerization or polycondensation have high permeability and a sufficiently large specific surface. A significant advantage of such materials is high porosity, which can reach $80 \%$ or more.
\end{abstract}

KEYWORDS: Porous composite material, Bulk porosity, Specific surface area, Precipitation, Thermal decomposition, Hydrothermal synthesis, Selective dissolution, Burning out, Fibers, Aerogels, Spinodal decay.

FOR CITATION: Kudryavtsev P.G. Main routes of the porous composite materials creation. Nanotechnologies in Construction. 2020;12(5): 256-269. Available from: doi: 10.15828/2075-8545-2020-12-5-256-269.

\section{INTRODUCTION}

$\mathrm{T}$ he porous material is a solid body containing in its volume free space in the form of cavities, channels, or pores, which determine the presence of an internal interfacial surface. Of note is the difference between the porous material and hollow containers. First, this difference lies in the fact that in porous materials, the average pore sizes $\left\langle\mathrm{D}_{\text {пор }}\right\rangle$ much smaller than the geometrical dimensions of the solid body:

$$
\left\langle\mathrm{D}_{\text {пор }}\right\rangle=\iiint_{V}^{m} D(x, y, z) \varphi(x, y, z) d V \ll<\sqrt{\bar{x}^{2}+\bar{y}^{2}+\bar{z}^{2}},
$$

where $\bar{x}, \bar{y}, \bar{z}$ - average body size $V$, along the axes. $D(x, y, z)-$ pore size at a given point in the body $V$. $\varphi(x, y, z)$ - three-dimensional probability distribution of the presence of pores at a given point in the body $V$.
Pores can be filled with gas or liquid. According to the classification of dispersed systems according to the state of aggregation of phases, porous bodies belong to dispersed systems with a solid dispersion medium and gaseous or liquid dispersed phases. Unlike porous materials, free-dispersed systems with a solid dispersed phase are peculiar reversed systems. If in the case of porous materials, a solid is a dispersion medium, in the second case, it is a dispersed phase.

The International Union of Pure and Applied Chemistry (IUPAC) has recommended the classification of porous materials according to average pore sizes. At the same time, they are divided into micro-, meso- and macroporous materials [1]. In the same way, with increasing dispersion, porous bodies are transferred from macroporous bodies to meso- and microporous bodies, in which the pore sizes are comparable with the sizes of molecules.

(c) Kudryavtsev P.G., 2020 
In the latter case, and the asymptotic limit, the idea of the inner surface begins to lose its physical meaning, by analogy with true solutions [2].

Macroporous materials are materials in which there are pores with a diameter of more than $50 \mathrm{~nm}$. Mesoporous materials are materials in which the structure is characterized by cavities or channels with a diameter in the range of $2 \div 50 \mathrm{~nm}$. Microporous materials have pores with a diameter of less than $2 \mathrm{~nm}$. Sometimes micropores are conventionally divided into thinner ultramicropores with a diameter of less than $0.7 \mathrm{~nm}$. Such pores are characteristic of zeolites [3], various adsorbents [4], and ionsieve inorganic sorbents and some other sorption materials [5]. The asymptotic variant of such pores can be considered defects of the crystal lattices of solids, such as solid solutions of subtraction. Subtraction solid solutions are formed based on chemical compounds in the crystal structure of which there are lattice sites that are not occupied by atoms of one of the components, i.e., vacancies are formed [6].

In the production of membranes and the implementation of the processes of membrane transfer of substances using a different classification. Membranes with a pore diameter of $<500 \mathrm{~nm}$ are considered microporous, and macroporous $>500 \mathrm{~nm}$ are microporous. This classification is due to differences in the mechanisms of transfer of molecules. In materials with a pore diameter much smaller than the mean free path of molecules, a hydrodynamic process, called the Knudsen flow, is realized. Under these conditions, the probability of molecules colliding with each other is less than the probability of their collision and reflection from the pore walls. In macroporous membranes, the transfer occurs through a viscous flow of liquid or gas.

Porous bodies in their structure are divided into corpuscular and spongy. The corpuscular porous bodies (for example, silica gels) consist of fused particles of different shapes and sizes, and the pores are the gaps between these particles and their ensembles. In spongy bodies (for example, porous glasses), it is impossible to isolate individual primary particles. The pores in them are a network of channels and cavities of various shapes and variable cross-sections. In most cases, the porous structure is set during synthesis and depends on the conditions of its implementation. For porous oxide bodies, the role is played by the type of solvent, the $\mathrm{pH}$ of the system, the calcination temperature, etc. It is possible to modify the materials even after their synthesis, leading to changes in the pore system. The main characteristics of porous bodies are porosity, pore size distribution, specific surface area. There are also open and closed porosity. The system of closed pores inside the body, in contrast to the open, does not communicate with the external environment.

This article is the completion of a series of articles on porous nanomaterials published in the journal "Nanotechnology in Construction" [39-43].

\section{General Methods for Obtaining Porous Materials}

The deposition is one of the most common methods for producing porous materials, usually accompanied by transitions: sol-gel-xerogel. Each of these transitions, be it a sol-gel, gel-xerogel, regardless of the mechanism of its action, makes a certain contribution to the overall process of pore formation. These transitions, as shown in [7], are very sensitive to external influences: the $\mathrm{pH}$ of the medium, the nature of the intermicellar fluid, the presence of surface-active substances, which dramatically affects the aggregation of particles with the formation of loose openwork spatial structures or paid formations. Therefore, reasonably acting on the flow of these processes, adjusting their depth and direction, you can control the structure formation, obtaining materials with a predetermined porosity and mechanical stability.

The preparation of the deposition of adsorbents, catalysts, inorganic ion exchangers has been studied in detail. To obtain them, the most used solutions of salts of mineral and carboxylic acids. The deposition can be carried out with aqueous solutions of ammonia, alkali, or soluble carbonates. The concentration, temperature, and deposition rate can be changed within the widest limits established empirically. Freshly precipitated hydroxides are usually amorphous (gels $\mathrm{MnO}_{2}, \mathrm{ZrO}_{2}, \mathrm{Nb}_{2} \mathrm{O}_{5}, \mathrm{Ta}_{2} \mathrm{O}_{5}$ ), and only some of them (gels $\mathrm{Al}_{2} \mathrm{O}_{3}, \mathrm{MgO}, \mathrm{TiO}_{2}$ ) crystallize with time, turning into cut particles [5]. The aging of the gel leads to a redistribution of the substance, because of which the particles approach each other and, in some cases, become larger, and their contacts grow together. This leads to the hardening of the skeleton of the gel, reducing its dispersion and, accordingly, reducing shrinkage during drying. Gels and xerogels can be divided by the type and shape of the particles that make them up. Their constituent particles are globular (silica gel and aluminum gel), rod-like (tungsten oxide gel), spindleshaped (barium sulfate), rod-shaped and filiform (vanadium pentoxide), etc. In practice, often used mixed materials, which in most cases have a synergistic effect [5].

Thermal decomposition, as a method used to obtain porous oxide materials by thermal decomposition of various compounds: hydroxides, carbonates, oxalates, hydrides, etc.

The resulting products are highly dispersed substances with a widely developed system of capillaries, sometimes quite definite in size and shape. Cases of the topotactic mechanism of decomposition are not uncommon when the structures obtained to retain the external dimensions and shape of the initial crystals with a strictly defined set of pores. The nature of the structure of such materials depends on the nature of the starting products and the reaction temperature. Also, this method is widely used to obtain active carbons. An extensive network of pores strongly depends on the degree of burnout of the carbon 
matrix, and with its increase, the volume of large pores increases [8].

By thermal decomposition of some polymers, coals with a very finely porous structure can be obtained. Examples include coal-based polyfuryl, formaldehyde, phenol-formaldehyde resins, polyvinylidene chloride, etc. [4].

Hydrothermal synthesis - is widely used to produce zeolites. To do this, a mixture of aluminum and sodium silicate is heated in an autoclave at a certain temperature. The porosity of the resulting materials depends entirely on the temperature and composition of the solutions. This method is widely used to modify the porous structure of gels, xerogels, and some natural aluminosilicates in the direction of large porosity [9].

The method of selective dissolution of substances. Selective dissolution of individual components of a substance using chemical reactions is one of the effective methods for creating or increasing porosity. This method is used to obtain a porous nickel catalyst (Raney nickel). For this purpose, an alloy of nickel with aluminum, containing approximately $50 \%$ of each component, is processed during cooling with a concentrated solution of sodium hydroxide, which dissolves aluminum. Similarly, Raney cobalt, Raney iron, etc., as well as porous glasses, can be obtained by treating two-component alkali-silicate or alkali-borate glasses [10].

Method of burning one of the components. The burning of the explosive component of hydrogels of various hydroxides is used to form the porosity of some sorption materials. This creates the possibility of creating a porous structure of a strictly defined size. Thus, porous silica gels with a unimodal micropore size distribution were obtained. The porous structure of this material, ASKM grade silica gel, was formed at the stage of burning carbon black from sodium silicate hydrogel at elevated temperature [11].

There are cases of using non-equilibrium plasma to create porous materials by burning off the organic matrix from the initial mixture. This technology is used to prepare for the analysis of biological samples, when necessary to remove organic matter, without changing the structure of inorganic components. The ability to remove carbon from the analyzed material without changing its remaining components (inorganic compounds) is used in chemical and crystallographic studies of the mineral components of coal.

The development of this method of synthesis of materials in modern conditions is template synthesis. Template synthesis refers to the synthesis of materials in the presence of agents controlling the structure. Template synthesis is a promising method for obtaining materials with a pre-defined, porous structure. The porous structure of the future porous material is set by selecting the appropriate template. The term "template" or a controlling agent structure is understood to mean additionally the materials or substances introduced during the syn- thesis that control the structure of the target material. A template is a central element surrounded by particles of a material; the removal of such a template creates a cavity with morphological and/or stereochemical functions like a template [12].

The possibility of preserving the structure (skeleton) at the macro and micro levels of inorganic material is remarkably interesting. It is enough $10-20 \%$ of mineral substances in the studied material to obtain a three-dimensional skeleton because of its oxidation in a nonequilibrium plasma [13].

\section{Methods of Forming Highly Porous Refractory Ma- terial}

There are two main ways of forming refractory ceramic products. The first way is the direct sintering of dispersions of ceramic fibers. This way is realized only with the use of high temperatures $\mathrm{T}=0.7 \cdot \mathrm{T}_{\mathrm{m}}$. Here, the $\mathrm{T}_{\mathrm{m}}-$ melting temperature of the material. In this case, in the temperature range $\mathrm{T}=(0.7 \div 0.9) \cdot \mathrm{Tm}$, rapid sintering is realized due to the evaporation of dislocations. Under these conditions, intense compaction of the material is observed due to the passage of the processes preceding melting. The processes associated with using a binder occur at significantly lower temperatures, corresponding to the transformation of components into stable chemical forms.

An example of obtaining material sintering methods is developed by NASA's Ames Research Center (USA); the process of obtaining the FRCI material is schematically depicted in Figure 1.

Initially, each type of fiber is subjected to conditioning. To ensure this process, micro-quartz fibers are placed in a plastic container with deionized water and hydrochloric acid, after which high purity nitrogen is blown in for 2 hours to remove soluble contaminants and non-fibrous inclusions. Then the micro-quartz fibers are washed three times for 5 minutes with deionized water.

For conditioning, Nextel-312 alumina-boron-silicate fibers are calcined at $1090^{\circ} \mathrm{C}$ for 90 minutes, which promotes the crystallization of their structure and reduces dispersity. The washed fibers of micro quartz and Nextel-312 are placed in a mixer, 2\% silicon carbide, and enough deionized water is added by weight. Then for a certain time, depending on the desired composition of the FRCI material and the desired density value, the dispersion is mixed in the mixer [16].

After mixing, the resulting dispersion of fibers is poured into a press, and the excess water is removed by the pressing method. As a result, receive a wet fibrous felt. Then the felt is slowly heated to 150 and maintained at a temperature of 18 hours. The felt obtained by the female is sintered at $1300^{\circ} \mathrm{C}$ for 90 minutes and cut to obtain the required parts. 


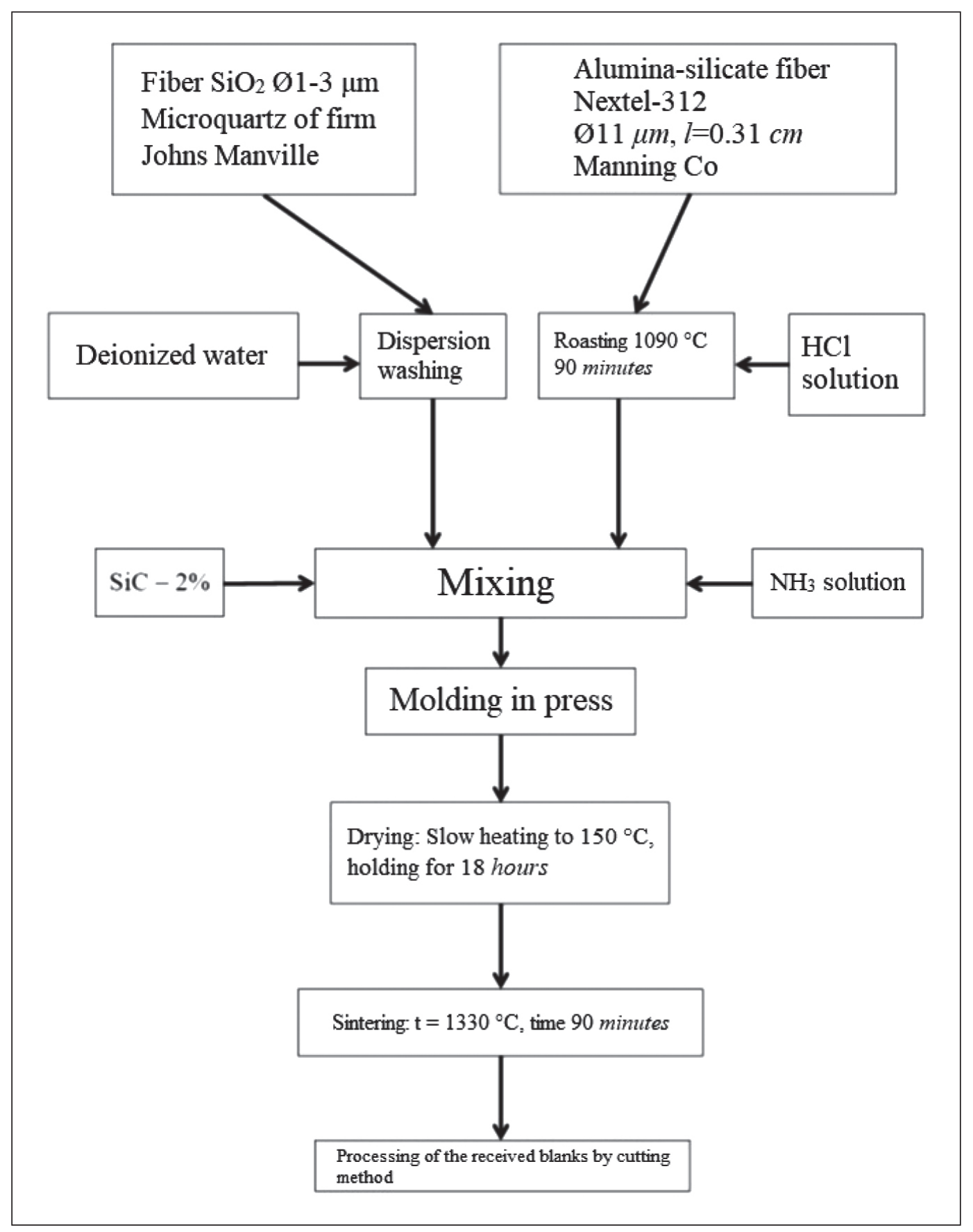

Fig. 1. The technological process of production of the material FRCI [16]

The second method is the use of a binder, which can significantly reduce the temperature of obtaining a porous product. The most technologically advanced and practical in the manufacture of fibrous material is the "wet method", i.e., the method of preparation of the suspension with a binder with the subsequent operation of formation.

According to [6], there are three basic binder dislocations within the fiber structure: segmental, agglomerate, and point. It seems that this classification can also be extended to compositions with an inorganic binder.

Segment structure corresponds to the placement of the binder in the fiber-based material in the cells formed by the mutual intersection of the fibers. An agglomerate structure is a random aggregation of a binder of various shapes. Some agglomerates do not participate in the work of the material since they are located between the sites of accumulation of fibers. Materials with an agglomer- 
ate structure are characterized by increased rigidity. The point structure corresponds to the distribution of the main amount of binder in the fibrous structure of the material in the form of individual points located at the intersections of the fibers. This structure corresponds to the most rational distribution of the binder with the maximum degree of its use.

The best-known binders in fibrous refractory ceramic materials are hydrated oxides of silicon and aluminum [14].

The technology for producing fibrous materials consists of the following operations: preparing an aqueous suspension of fibers, introducing a solution of a binder into the suspension in an amount necessary to bind the fibers into a strong frame, precipitate a binder in the form of an aluminum hydroxide dispersed phase with an ammonia solution to obtain a uniform suspension concentration, dehydrating it with prepressing to a given volumetric weight and drying the material, followed by firing.

However, the processes of precipitation of aluminum hydroxide with the help of precipitating agents lead to the formation of an uneven solid hydroxide phase, which complicates its uniform distribution in the volume of the suspension of fibers. A known method for the homogeneous production of hydrated alumina by the temperature hydrolysis of certain substances of precipitating agents [15]. Such substances includehexamethylenetetramine, biuret, urea. The most common of these is urea $\mathrm{CO}\left(\mathrm{NH}_{2}\right)_{2}$. The processes of homogeneous precipitation of hydrated alumina are widely used to produce sorbents and supports for catalysts. This method is well studied and may be of interest tointroducing a binder in the preparation of fibrous materials.

A method of obtaining a heat-shielding material by applying hydro mass on specific areas of the product. Similar material was developed based on alumina fibers and aluminosilicate fibers with an inorganic binder. The material has a high enough heat resistance $-1470 \div 1870 \mathrm{~K}$ (depending on the type of fibers), good physical and mechanical characteristics with an apparent density of $0.30 \div 0.34 \mathrm{~g} / \mathrm{cm}^{3}$.

\section{Porous Aerogels Based Nanocomposites}

Composite materials are usually obtained by combining two different materials. In a general sense, the creation of composites is used to take advantage of each type of material and to minimize their disadvantages. For example, silica aerogels are fragile substances. Simultaneously, another component in the resulting material can increase the strength of the material, which, in turn, has the desired optical properties, high surface area, and low density as in silica aerogel.

In addition to these synthesis and processing methods, it is necessary to emphasize that the flexibility of the sol- gel process allows us to increase the choice of aerogels other than silicon dioxide, aerogels based on other materials that are currently available. The architecture of massive materials can be adapted using template methods. The chemistry of the gel can be changed by grafting, either during the gelling process or after gelling [17].

Composites and nanocomposites can be created by impregnating foams or fiber networks, dispersing particles, powders, or polymers, or by synthesizing silica-based mixed oxides, or oxides of other metals. Organic hybrids of silica can also be made by many methods, such as cogelling and cross-linking or by interacting with functionalized particles $[5,18]$.

In recent years, a large amount of research has been carried out to obtain energy materials. There are works on using materials based on aerogels and sol-gel derivatives to obtain nanostructured energy composites (for example, explosives, rocket fuel, and pyrotechnic compositions). Their characteristics have been studied. Aerogels have a unique density, composition, porosity, and particle size, as well as low temperature and mild conditions of chemical synthesis methods, all of which make them attractive as candidates for creating energy nanomaterials [19].

The use of these materials and methods in this field of technology has led to three main types of sol-gel energy materials [20]:

1. Pyrotechnics - inorganic sol-gel oxidants / metallic fuel (thermite composites).

2. Sol-gel derivatives of porous pyrophoric metal powders and films.

3. Organic sol-gel fuel / inorganic nanocomposite oxidizers (composite solid rocket fuels and explosives).

The behavior of all sol-gel nanostructured energetic materials largely depends on several factors, including surface area, the degree of mixing between phases, the type of mixing (sol-gel or physical mixing of solids), the method of loading solids, and the presence of impurities. Sol-gel methods are attractive for the field of obtaining nanostructured energy materials. These methods offer many variations in the shape of the materials produced, such as monoliths, powders, and films, and have a wide compositional versatility. These attributes, combined with the rigor of the synthetic control of the microstructural properties of the sol-gel matrix, provide the preparation of energy nanocomposites with re-tunable characteristics.

For the past two decades, the field of nano research has been one of the most active areas of research in various scientific disciplines, and energetic materials were no exception to this [21]. Using nanomaterials and advanced manufacturing techniques, energy nanocomposites have been synthesized, which have promising opportunities. Energy nanocomposites are defined as mixtures of an oxidizer and fuel whose particles have dimensions or at least one critical size less than $100 \mathrm{~nm}$. Reducing the size increases the contact surface area between the phases of the 
reactants. This has been achieved using various methods, including methods of vapor condensation, micellar synthesis, chemical reduction, ultrasonic mixing, and mechanical methods of mixing. Particularly good results were obtained. For example, for pyrotechnic nanocomposites $\mathrm{Al} / \mathrm{MoO}_{3}$, burning rates were recorded by almost three orders of magnitude greater than those of conventional mixtures. Also, properties of energetic materials, such as sensitivity to impacts, depending on the size of the particles. Energy materials with smaller particle sizes may be less sensitive to ignition and have better safety properties [23]. These two important examples provide a powerful incentive for the application of nanomaterials and technologies in the energy field. Aerogels and other gel-like materials derived from sols have been investigated in the last decade as nanostructured energy materials.

Along with good miscibility, energy nanocomposites have extremely high interfacial areas; the sol-gel method for producing these materials allows for even larger interfacial contact zones. All these favorable attributes have led to active research on applying sol-gel chemistry to the research and development of energy materials.

Organic-mineral nanocomposites, based on silica aerogels, possess a complex of unique optical properties. The refractive index of an aerogel modified with trimethylsilyl groups may be in the range of $1.008 \div 1.060$, depending on their densities. Figure 2 shows the relationship between the density and the refractive index of an aerogel based on silicon oxide modified with trimethylsilyl groups. The relative value of the refractive index $\boldsymbol{n}$ is almost proportional to the density of the aerogel material in the high porosity range. This result is consistent with the theory, namely the Maxwell-Garnet ratio, applied to nanocomposites composed of organically modified silica and air [24].

Since the modified aerogels have excellent optical properties, transparency, extremely low index of refractive index, and moisture resistance, they were often used as a medium in Cherenkov counters. When a charged particle passes through a transparent medium at speed faster than the speed of light in the material, Cherenkov luminescence occurs.

Although the monolithic aerogel blocks of silicon dioxide, produced by supercritical drying methods, are rather expensive for industrial applications, they have contributed to the progress in such scientific fields as high-energy physics. The progress of science has always contributed to the improvement of research and development in the industrial world, so we can expect that aerogel can become a pioneer of technologies such as nanocomposites, optics, space research, energy devices, and so on $[20,26]$.

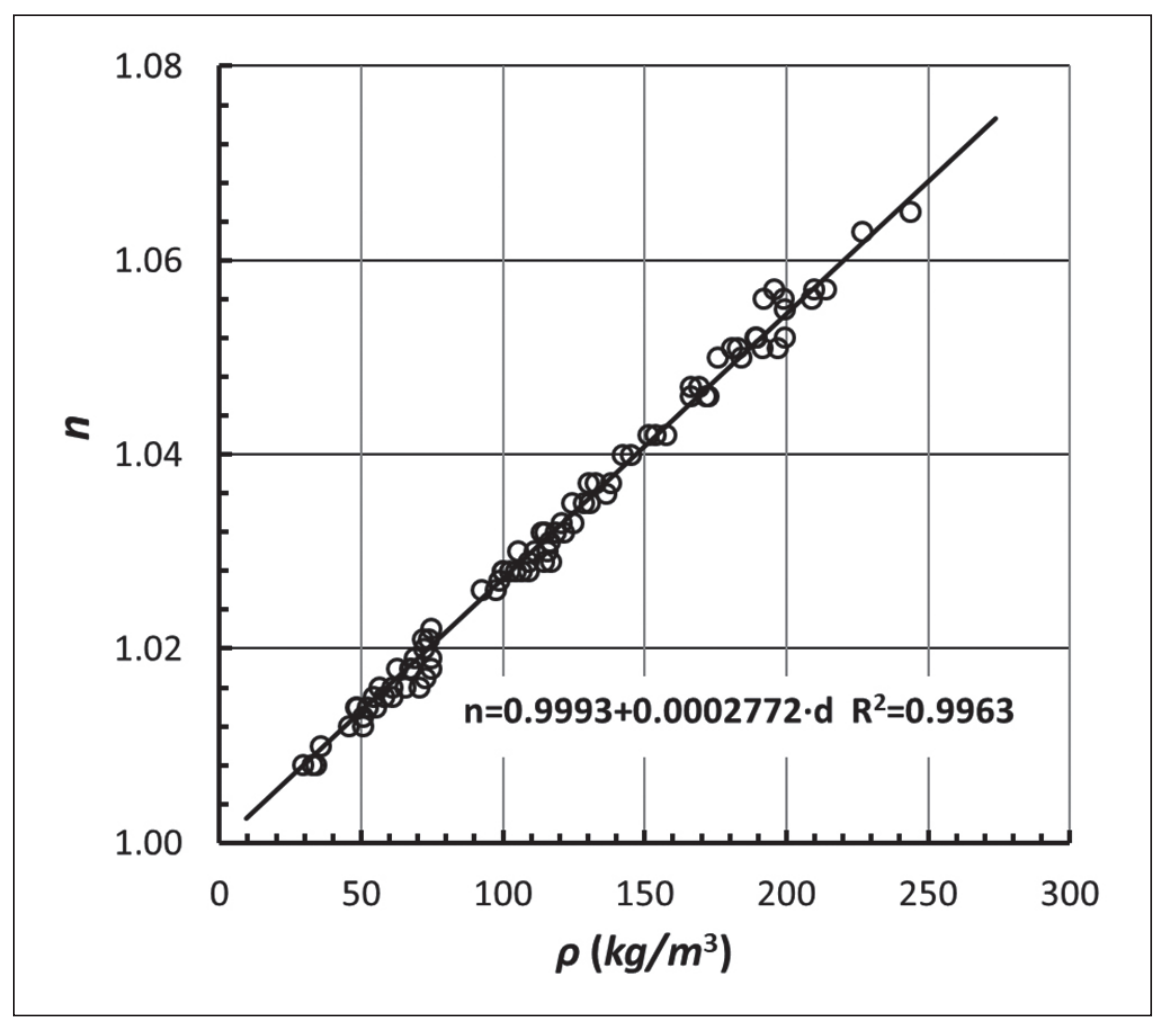

Fig. 2. Correlation between density and refractive indices of silica hydrophobic aerogels [25]: $n$ - refractive index; $\rho-$ silica aerogel density $\left(\mathrm{kg} / \mathrm{m}^{3}\right)$ 
Active work is underway in nanoscale engineering of silica-based composites to create various sensors. In work [27], they have described "silica composites modified silica" prepared after modifying the silica gel during gelation, catalyzed by the base, with another silica sol, this time obtained using acid catalysis. This basecatalyzed acid-modified gel is then treated using carbon dioxide supercritical extraction to obtain an aerogel. The aerogel monoliths obtained because of this process have bulk properties of silica aerogel obtained in the main catalytic process, including good transparency. In contrast, the surface properties are more typical of the aerogel obtained by acid catalysis. Consequently, it is possible to capture various types of highly polar molecules, including acid-base indicators, and use them as an interface of inappropriate sensors.

In [27], reported on composite aerogels of silicon dioxide containing metallic colloidal particles (gold or platinum) and that had optical transparency of silica aerogels in combination with the surface and optical properties of a metallic colloid. Metallic colloidal particles are evenly distributed throughout the entire volume of the mixture and, therefore, are isolated from each other. Simultaneously, the porosity of the silica matrix makes these metallic colloid particles available to particles that pass through the matrix. The surface of the metal colloid can be modified either before or after gelation, to adapt it to the optical properties of the material.

Preliminary results are described in [28], which demonstrate the inclusion of polyaniline nanofibers in silica aerogel prepared based on tetraethoxysilane, and carbon dioxide supercritical extraction leads to an increase in the strength of materials. In this case, it becomes possible to use them to detect gaseous acids and bases potentially. It was found that including the introduction of only about $6 \%$ polyaniline by weight of the material, the strength of the aerogel was increased three times, upon receipt of the same low density $\left(0.088 \mathrm{~g} / \mathrm{cm}^{3}\right)$. When using gold electrodes on the surface of a composite aerogel, a sharp decrease in resistance was observed when the aerogel was exposed to $\mathrm{HCl}$ vapor.

After carrying out the entire stages of the synthesis process, the aerogel is a solid, amorphous, but extremely porous (75-99\% porosity) material. The last step in the transformation is its compaction by heat treatment. It is often necessary to transform the material by sintering the aerogel into solid glass devoid of porosity, with a relative density of 1 . Relative density - the ratio between the bulk density of aerogel and the density of silica glass $\left(2.2 \mathrm{~g} / \mathrm{cm}^{3}\right)$. Figure 3 shows a typical evolution of relative density and specific surface area during sintering by heat treatment $[20,27]$. These curves strongly depend

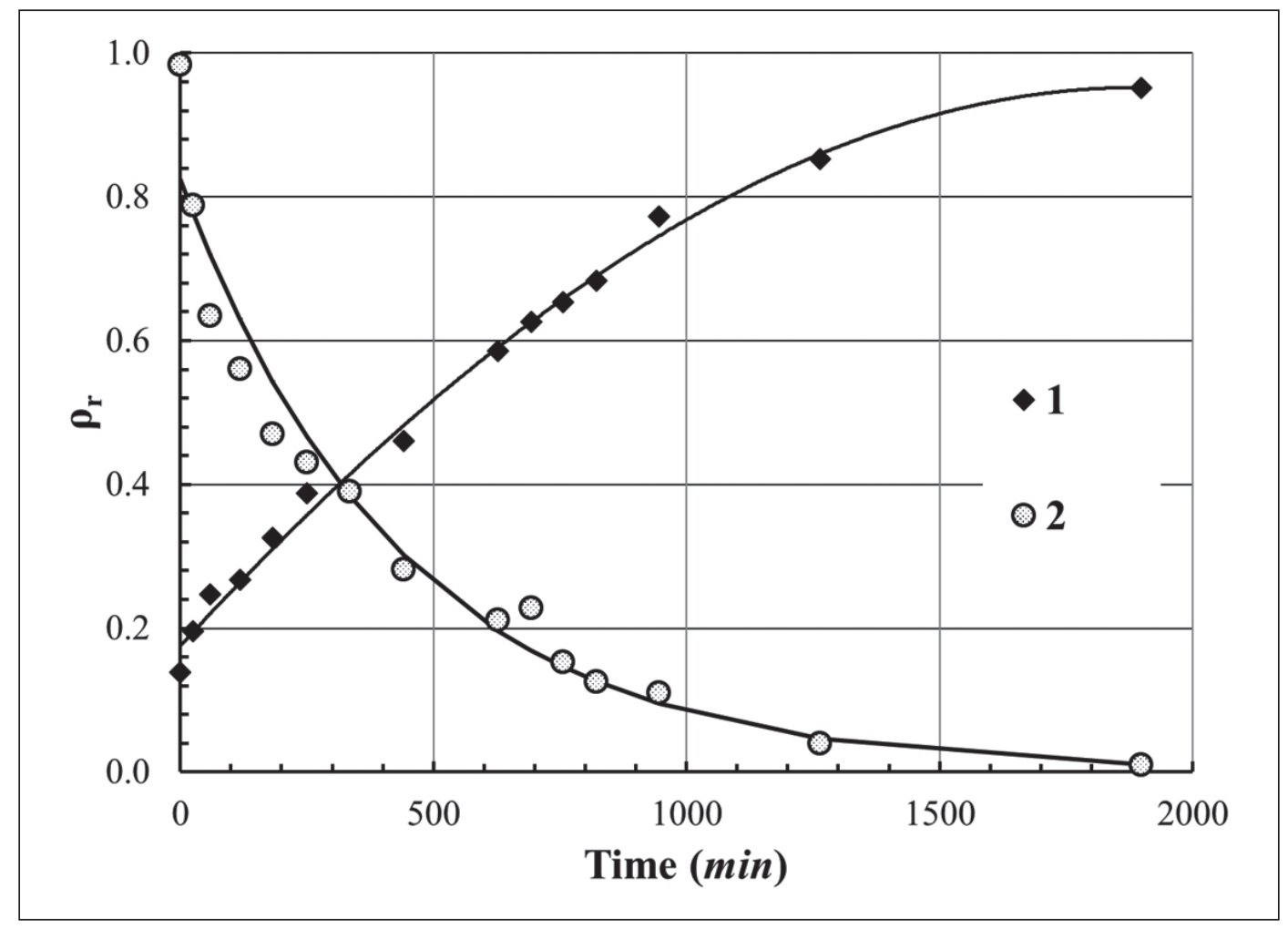

Fig. 3. The evolution of aerogel relative density $\rho_{\mathrm{r}}(1)$ and specific surface $S(2)$ as a function of sintering time at $1000^{\circ} \mathrm{C}[25]$ 
on the heat treatment temperature and on the content of hydroxyl groups in the aerogel structure, which affects the viscosity of the aerogel [27].

Gels that are initially non-crystalline may crystallize during the subsequent heat treatment. The successful formation of glass results from competition between phenomena that lead to compaction and those that contribute to crystallization.

These data follow such an important use of nanocomposites based on aerogels of silicon oxide, which is the sealing of radioactive waste from nuclear power plants [29]. Actinides and other radionuclides generated in the nuclear fuel cycle, when reprocessing spent fuel, are represented as salts in aqueous solutions. Using the fully open porous structure of the aerogel, it is possible to fill the entire volume of the aerogel with solutions of these salts. Then the liquid phase is removed by evaporation, and the porous composite material (aerogel + salt) is completely sintered, which leads to the synthesis of a multi-component material. The porous structure of the aerogel is used as a receiving volume. Following the small pore size of the aerogel, the preparation of such a nanocomposite is an amazingly simple process. The size of the domains will depend on the size of the pores in the aerogel and on the content of actinides in the solution.

However, if trying to fill an aerogel with a liquid such as water, capillary forces may destroy aerogel [30]. Due to the complexity of the texture of the aerogel, it is difficult to make a detailed calculation of local stresses when it is filled with liquid; it depends on the surface energy of the liquid-vapor and the size of the pores. This phenomenonis called decrepitation. This term originated in the mining industry as a selective rock opening based on the ability of individual minerals to break down along the cleavage planes when heated. It then rapidly cooled or only when heated. The process of decryption destruction is explained by the presence of crystallization water, gas-liquid inclusions, low thermal conductivity, and thermal expansion coefficients of individual minerals, pronounced cleavage. Heterogeneous thermal properties of the components of the mineral during heating also lead to the appearance of many sources of internal stresses. The increase in internal stresses is achieved by a sharp cooling of the surface of the pieces of the mineral, which leads either to its destruction or to a weakening of the bonds between the minerals. A similar phenomenon is observed in microporous materials when removing the liquid phase from the pores. In this case, because of the action of capillary forces, high internal stresses arise. If these stresses exceed the strength of the framework of the porous material itself, then its destruction occurs.

Thus, to avoid cracking the material during filling, various strategies can be proposed:

1. Aerogel synthesis with large pores that will reduce the magnitude of the capillary forces.
2. Improving the mechanical strength of the aerogel due to its partial aging and sintering.

3. Surface functionalization by grafting chelating groups to the aerogel surface.

\section{Production of Porous Materials Using the Phenom- enon of Spinodal Decomposition}

Special materials, the structure of which is formed in the process of microphase separation during polymerization or polycondensation, have high permeability and a sufficiently large specific surface. A significant advantage of such materials is high porosity, which can reach $80 \%$ or more. Historically, the first materials of this type were ion exchange resins. The size of the spherical particles of the resin is a few micrometers, and the pore diameter is $10 \div 120 \mathrm{~nm}$. Recently, many publications on the synthesis of polymer macroporous monoliths, which are used in various fields of chromatography, have appeared. The basic theoretical positions are formulated, and the techniques are developed, allowing them to change and control their structure over a wide range. However, the low chemical resistance and a heat resistance of polymers somewhat narrow the range of their application. The most detailed review of the methods of synthesis of such materials is made in [30].

Macroporous oxide materials obtained by the sol-gel method are distinguished by high heat resistance [30]. By changing the composition of the reaction mixture, it is possible to form both spongy and particulate oxide blocks of large size containing macropores with sizes ranging from 0.05 to $100 \mu \mathrm{m}$. Thus, monolithic materials based on oxides of silicon, aluminum, zirconium, and titanium, resistant to water vapor and high temperatures, were synthesized.

Phase separation processes in polymerizing systems are extremely sensitive to minor changes in the synthesis conditions. This effect is due to the non-equilibrium of these processes. The non-equilibrium of processes often leads to the non-reproducibility of the results obtained and the wide distribution of pores in size. Such defects are deprived of template synthesis of macroporous systems. The formation of sol with the subsequent formation of a gel, in this case, occurs in the free pore space of the template. Polymeric microspheres, natural materials, and even ice microcrystals can be used as templates [31]. Materials synthesized using templates have a highly ordered structure, which leads to the emergence of new properties and expands their field of application.

Compared with organic polymers, inorganic oxide materials have higher strength, thermal stability, and chemical resistance, which significantly expands the scope of their application. High-purity and homogeneous oxide materials can be obtained using sol-gel synthesis, which controls the structure of products at all stages of the pro- 
cess [32]. Sol-gel processes occur in polar solvents on a both aqueous and organic basis. Solutions of various salts and alkoxides of metals can be used as precursors. When using alkoxides, the final material can be obtained in a purer form without impurities of alkali metal ions.

In the sol-gel transition process, the growth of metal oxide oligomers occurs, which form the network of the gel. After drying and heat treatment of the gel, it is possible to obtain amorphous and crystalline oxide materials in the form of films, fibers, or powders. A feature of the sol-gel method is the formation of pores in the gel phase, filled with solvent. Therefore, bulk samples of gels during drying can crack under the action of capillary pressure forces. To obtain monolithic blocks, it is necessary to carry out this operation in liquids with low surface tension or under supercritical conditions. This produces nanoporous materials, called aerogels, whose porosity can reach $90 \%$.

The idea of using sol-gel technology to obtain macroporous oxide materials was born in the early 90 s of the last century. During the synthesis of macroporous monoliths based on zirconium and titanium oxides, problems arose since the hydrolysis reactions of titanium and zirconium alkoxides proceed at high rates. As a result, the phase decomposition in the system proceeds via the nucleation mechanism, which results in the production of dispersed oxides, sometimes of a spherical shape. At present, methods have been developed to synthesize macroporous block materials based on oxides of titanium, aluminum, and zirconium [33].

The successive stages of the synthesis of macroporous monoliths and the processes occurring at each stage can be considered using the example of the hydrolysis of silicon alkoxides. The hydrolysis of alkoxides is carried out in a solvent (usually water), a catalyst, and additives, such as water-soluble polymers. An open porous structure is formed by passing phase separation in a system containing alkoxide oligomers, a solvent, and a pore former, which in most cases, is a water-soluble polymer. The synthesis of monolithic macroporous samples requires that the system formed because of phase separation contains two spatially continuous phases: the gel phase and the poreforming phase. Only in this case, after removing the blowing agent, the formation of open macropores, penetrating the entire volume of the monolith, is possible.

Consider the mechanism of phase separation processes during sol-gel synthesis. The change in the free energy of a binary system containing at least one type of polymer molecule can be described by the Flory-Huggins equation [30, 34]:

$$
\Delta G \propto R T\left(\left(\Phi_{1} / \mathrm{P}_{1}\right) \ln \Phi_{1}+\left(\Phi_{2} / \mathrm{P}_{2}\right) \ln \Phi_{2}+\chi_{12} \Phi_{1} \Phi_{2}\right),
$$

where $\Phi_{i}$ and $\mathrm{P}_{i}(i=1,2)$ - respectively, the volume fraction and degree of polymerization of each component; $\chi_{12}$ - the interaction parameter between the components. The first two terms in brackets express the entropy contribution to the free energy, and the last is enthalpy.

With a decrease in the absolute value of the negative entropy component, the system destabilizes, i.e., an increase in the degree of polymerization of any of the components makes the mixture less compatible. The magnitude of the enthalpy component also depends on the molecular weight of the inorganic polymer. Because of the polycondensation of an inorganic polymer, the amount of hydroxyl groups in its polymer particle decreases. This, in turn, causes a decrease in the interaction of the inorganic polymer with water molecules or a blowing agent. Thus, the initial single-phase system, with an increase in the molecular weight of the polymerizable component, becomes less stable. As a result, microphase separation occurs.

By analogy with the processes that occur with decreasing temperature in chemically non-reacting systems, the process of polycondensation, leading to a decrease in the mobility of monomer molecules during the formation of chemical bonds between them, as defined in as "chemical freezing". This circumstance makes it possible to use the classical thermodynamic approach developed by Gibbs and study microphase separation in cured polymer-oligomeric systems concerning sol-gel systems [34].

On the concentration dependence of the free energy $G(\Phi)$, there are inflection points at which the sign of the second derivative changes $\partial^{2} G / \partial \Phi^{2}$. If $\partial^{2} G / \partial \Phi^{2}>0$, the diffusion fluxes of the components are directed towards the reduction of composition fluctuations, whereas as $\partial^{2} G / \partial \Phi^{2}<0$ the circulation of diffusion flows occurs and the so-called "ascending diffusion" arises. If the intensity of fluctuations increases, then the single-phase system becomes unstable and should spontaneously disintegrate into regions having an equilibrium composition. The region of the instability of a single-phase system is limited in the phase diagram of a spinodal (Figure 4). On the "outer" side, a region of metastable states adjoins the spinodal, bounded by the binodal, on which the first derivative $\partial G / \partial \Phi=0$. On the ordinate axis, a conditional value equivalent to the temperature and the inverse of the molecular weight of the siloxane oligomers is plotted.

In the region between the binodal and spinodal, the diffusion fluxes of the components in the system are directed towards a decrease in the composition fluctuations. In this case, an energetically unfavorable effect of forming an interface between the phases leads to the fact that the system is unstable only concerning fluctuations whose dimensions are larger than the critical value. The fluctuations in the composition are close to the new phase. Such fluctuations are called critical germs. The resulting critical nuclei increase in size, and 


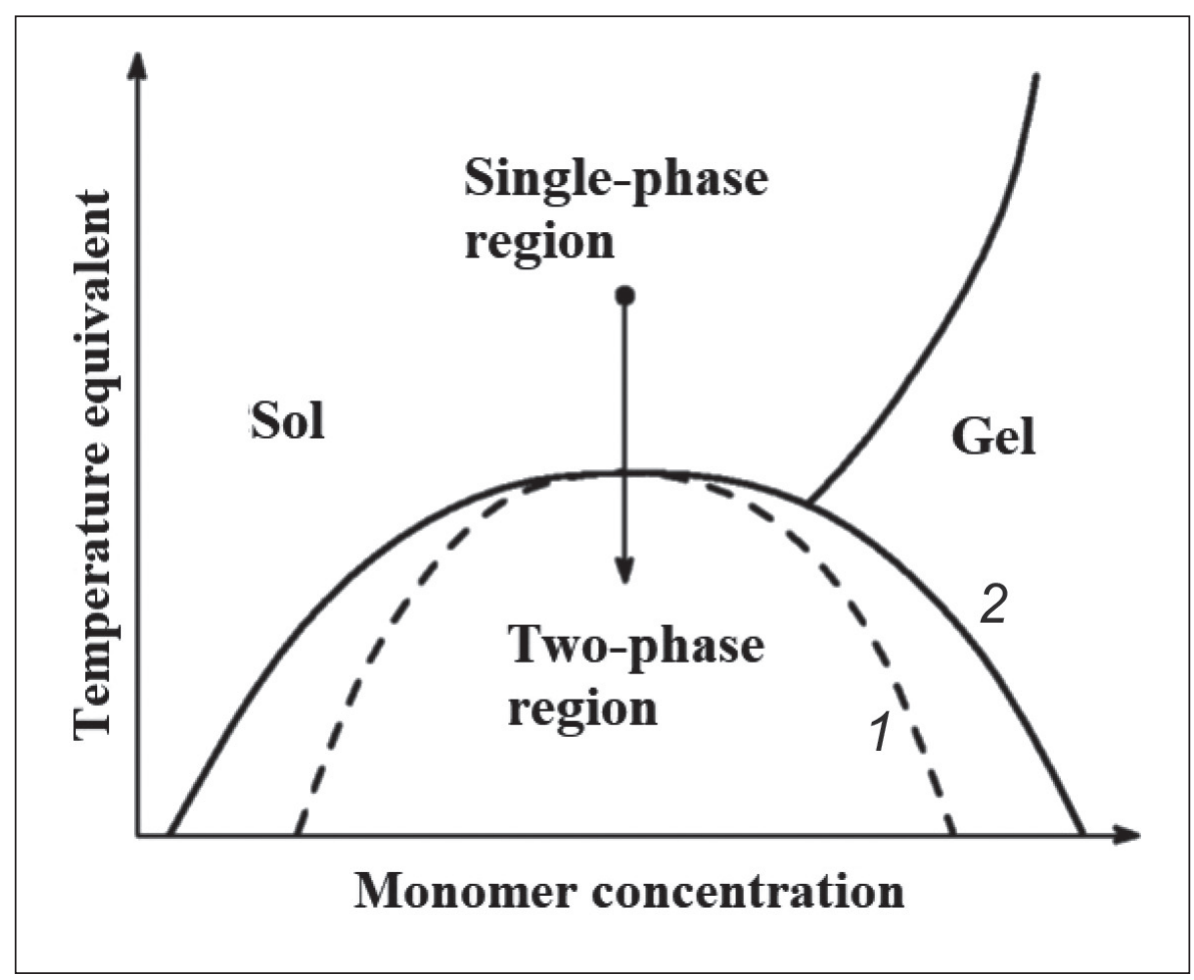

Fig. 4. Phase diagram of the gel-forming system [94,106]: 1 - spinodal, 2 - binodal

the separation of the phases is carried out according to the nucleation and growth mechanism.

Sol-gel systems, the phase decomposition of which is carried out by such a mechanism, are dispersed in one of the components, making it impossible to obtain macroporous blocks and monoliths on their basis. Near the critical point of two-phase equilibrium, the region of metastable states narrows, contracting to a point. This circumstance creates favorable prerequisites for achieving unstable states of the system using the near-critical transition through the binodal and spinodal, which in Figure 4 is depicted by the arrow.

Caught in an unstable state, the system quickly loses spatial homogeneity, and relaxing is a particularly modulated structure. By spinodal decay, we understand the development of a continuous inhomogeneous structure, which is a consequence of thermodynamic instability. A similar model structure is depicted in Figure 5 [34]. Spinodal decomposition occurs in areas of high concentrations of both components. A sign of phase separation by a spinodal mechanism is the appearance of diffraction maxima on the small-angle $\mathrm{X}$-ray scattering curves.

The transverse size of individual domains at the initial stage of spinodal decomposition depends on the rate of transition of the system to an unstable state and does not exceed $100 \mathrm{~nm}$. Subsequently, inhomogeneities can be enlarged without losing spatial continuity and reaching a size of $1 \mu \mathrm{m}$ or more or disintegrate into separate formations. Studies of the spinodal decay kinetics [35] make it possible to trace the process of formation, enrichment, and in some cases, the coalescence of microregions of the emerging structure.

Spinodal decomposition in a system containing siloxane oligomers leads to the formation of two spatially continuous phases enriched with oligomers, and the other is depleted of them. In parallel with the phase separation in the system, the formation of gel crosslinks occurs, leading to a decrease in the mobility of the components. This allows you to fix the bicontinuous structure and provides the possibility of obtaining on its basis of open-cell monolithic materials. The final morphology of the material is determined by the kinetics of coalescence of microregions formed in the process of spinodal decomposition, and the kinetics of the sol-gel transition. The structural parameters of porous monoliths are monitored during synthesis using small-angle $X$-ray scattering, scanning electron microscopy, and laser scanning confocal microscopy [36].

The maturation of the gel should be carried out in a sealed vessel at a constant temperature. In the process of maturation, continue the reaction of hydrolysis and polycondensation. The course of these reactions in the gel is slower and leads to additional crosslinks in the gel network. The time required for the gel to mature is usually several times longer than the gel time. The sign of its end 


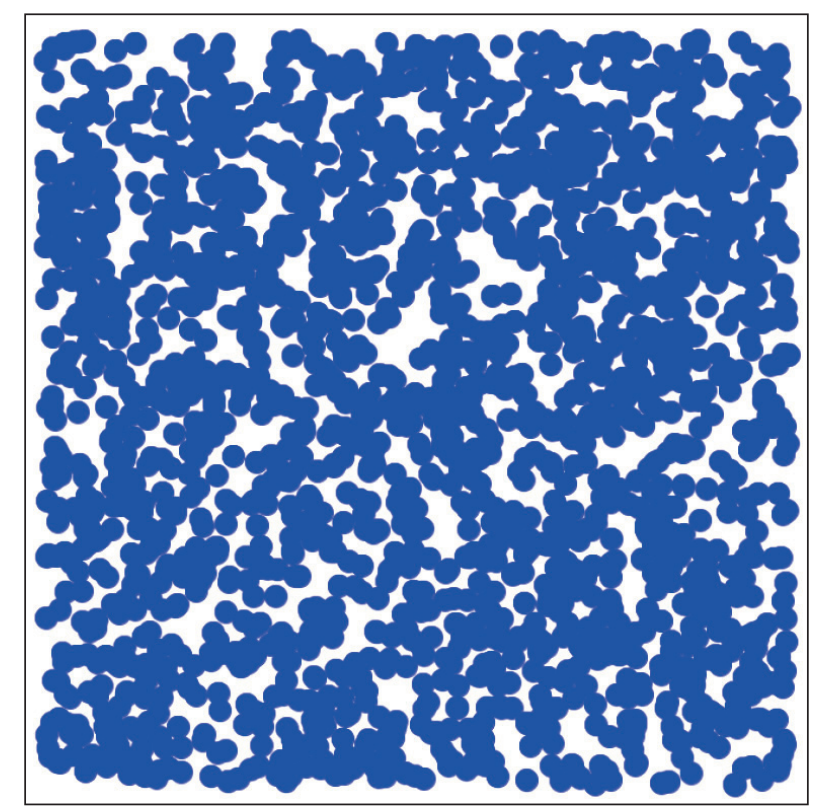

Fig. 5. A cross-sectional view of the structure formed by the mechanism of spinodal decomposition [30, 34]. The dark area is gel, the light area is sol

is the shrinkage of the material and the release of a separate solvent phase. To obtain pores with a narrow size distribution, the wet gel must be well matured.

After the gel has matured, the solvent replacement process begins. This is the next stage of the formation of macroporous blocks. This replaces the solvent, which is carried out when the gel is still wet. The essence of this process consists of immersing the gel in an external solvent, which differs from that added in the synthesis process. An aqueous solution of ammonia is often used as an external solvent since the alkaline medium helps to accelerate the polycondensation reaction, increase the strength, and modify the meso- and microporous structure of the gel. The structure of the gel can change in the process of dissolution under the influence of an external solvent, in which amorphous silica has substantial solubility. In this case, the redeposition of silicon oxide on the surface of the gel network can occur. Therefore, increasing the $\mathrm{pH}$ of the external solvent is the easiest way to increase the average pore size while maintaining the same width of the pore size distribution [37].

Instead of replacing the solvent with aqueous ammonia, hydrothermal treatment is sometimes used. In the process of hydrothermal treatment at elevated temperatures and high $\mathrm{pH}$ values, substance transfer and overgrowth of the contact points of gel particles occur, which makes the structure more durable and avoids cracking of the samples during drying under normal conditions. A variant is possible with the introduction of urea at the initial stage of sol-gel synthesis, followed by heating the material obtained to a temperature above $80^{\circ} \mathrm{C}$ after its maturation. At this temperature, urea decomposes with the release of ammonia.

Drying of wet gel samples is carried out in an oven with air circulation, while volatile components slowly evaporate. The required temperature varies from 40 to $80^{\circ} \mathrm{C}$, depending on the solvent used.

To obtain monolithic bulk samples, drying is a critical step. The Young-Laplace equation expresses the dependence of the capillary pressure $(P)$ in a cylindrical pore on the surface tension $(\gamma)$ at the liquid-vapor interface, wetting angle $(\theta)$, and pore radius $(r)$ :

$$
\mathrm{P}=(2 \gamma \cos \theta) / r
$$

In small pores, the capillary pressure is high; therefore, drying of gels with pores measuring at the level of nanometers is recommended under supercritical conditions, when there is no liquid-vapor interface. The capillary pressure in the macropores is much less, and with slow evaporation of the solvent, the macroporous gels withstand drying under normal conditions. It should be noted that the violation of the integrity of the sample during the drying process is caused not so much by the presence of capillary pressure, as by the appearance of a pressure gradient, and with an increase in the linear dimensions of the gel, the risk of cracking increases significantly. High molecular weight pore-forming agents remaining in the pores even after the solvent replacement procedure provide some additional elasticity of the gel and con- 
tribute to preserving its integrity. Linear shrinkage of the samples during ripening and drying is $50 \div 70 \%$. In this case, the pore size is proportionally reduced.

The final stage of the preparation of monoliths is heat treatment, which ensures the decomposition of organic components. Heating of materials to temperatures above $1000^{\circ} \mathrm{C}$ and their cooling is carried out at a rate of $\sim 100 \mathrm{deg} / \mathrm{h}$. In the process of heat treatment and the removal of organic components, the final modification of the structure occurs. Heating materials to a temperature of $600^{\circ} \mathrm{C}$ causes evaporation of nanoscale pores and decreases the proportion of mesopores. When heated above $1000^{\circ} \mathrm{C}$, the mesopores disappear, and porous glasses are obtained.

For sol-gel technology, in contrast to the technology for producing macroporous polymers, there are no general regularities relating to the influence of the synthesis conditions and the concentration of one or another component on the final gel structure. Since acid-base interactions in the system cause hydrolysis and polycondensation, the pore-forming agent is not an inert substance in the full sense of the word. It can exchange ions with a solution, which is typical, for example, of sodium polystyrene sulfonate or polyacrylic acid, and adsorb on the surface of siloxane oligomers, like polyethylene oxide, or undergo hydrolysis, like formamide. Therefore, the concentration dependences of the size of macropores appear peaks associated with changes in the structural characteristics of the monolith, the formation of spherical particles in macropores, etc. To date, the systems in which polyethylene oxide with a molecular weight $10^{4} \div 10^{5}$. It was shown [38] that for polyethylene oxide with a molecular weight equal to $3.5 \cdot 10^{4}$, an increase in the system of molar ratio polyethylene oxide: $\mathrm{SiO}_{2}$ in the system from 0.5 to 0.7 leads to a decrease in pore size from 10 to $0.2 \mu \mathrm{m}$. An increase in the molecular weight of the polymer also leads to a decrease in pore size.

\section{CONCLUSION}

Porous materials are solids, containing in their volume free space in the form of cavities, channels, or pores, which determine the presence of an internal interfacial surface.

A review of the main methods of obtaining porous materials. The deposition is one of the most common methods for producing porous materials. Thermal decomposition of various compounds is also used as a method to obtain porous oxide materials. Hydrothermal synthesis is widely used to produce zeolites. The method of selectively dissolving substances using chemical reactions is also one of the effective methods for creating and increasing the porosity of materials. The burning of the explosive component in the hydrogel phase of various hydroxides is also used to form the porosity of some sorption materials.

The paper presents an overview of the methods for forming highly porous refractory materials. There are two main ways of forming refractory ceramic products. The first way is the direct sintering of dispersions of ceramic fibers. The second method is the use of a binder, which can significantly reduce the temperature of obtaining a porous product.

The analysis of methods for obtaining porous nanocomposites based on aerogels is presented. Typically, composite materials are obtained by combining two different materials. In general, the creation of composites is used to take advantage of each type of material and to minimize their disadvantages. For example, aerogels are fragile substances. Simultaneously, another component in the resulting material can increase the strength of the material, which, in turn, has the desired optical properties, high surface area, and low density as in silica aerogel.

A new method for obtaining porous materials is the use of the phenomenon of spinodal decomposition. High permeability and a sufficiently large specific surface are possessed by materials whose structure is formed in microphase separation during polymerization or polycondensation. A significant advantage of such materials is high porosity, which can reach $80 \%$ or more.

Spinodal decomposition in a system containing siloxane oligomers leads to the formation of two spatially continuous phases enriched with oligomers, and the other is depleted of them. Parallel to the phase separation in the system, the formation of gel crosslinks occurs, leading to a decrease in the mobility of the components, which makes it possible to fix the bicontinuous structure and makes it possible to obtain on its basis open-cell monolithic materials. The final morphology of the material is determined by the kinetics of coalescence of microregions formed in the process of spinodal decomposition, and the kinetics of the sol-gel transition.

\section{REFERENCES}

1. Everett D.H. Manual of Symbols and Terminology for Physicochemical Quantities and Units: Appendix II: Definitions, terminology and symbols in colloid and surface chemistry. Part 1: Colloid and surface chemistry. Pure Appl. Chem. 1972;31: 577-638. 
2. Kralchevsky P.A., Danov K.D., Denkov N.D. Chemical Physics of Colloid Systems and Interfaces. Chapter 7. In: Birdi K.S.(eds.). Handbook of Surface and Colloid Chemistry. CRC Press; 2008. Available from: https://doi. org/10.1201/9781420007206.

3. Breck D. Zeolite molecular sieves. Moscow: Mir; 1976.

4. Komarov B.C. Adsorbents and their properties. Minsk: Science and Technology; 1977.

5. Kudryavtsev P.G. Alkoxides of chemical elements - promising class of chemical compounds which are raw materials for Hi-Tech industries. Scientific Israel - Technological Advantages. 2014;16(2): 147-170.

6. Gusev E., Garfunkel E., Dideikin A. (Eds.) Advanced Materials and Technologies for Micro/Nano-Devices, Sensors and Actuators. Series: NATO Science for Peace and Security Series B: Physics and Biophysics. Springer; 2010.

7. Ermolenko N.F., Efros M.D. Regulation of the porous structure of oxide sorbents and catalysts. Minsk: 1971.

8. Belikova S.E. Water treatment: Handbook. Moscow: Aqua-Term; 2007.

9. Neimark I.E., Sheinfine R.Yu. Silica gel, its properties and production. Kiev: 1973.

10. Kerber M.L. Polymer composite materials. Structure. Properties. Technology. SPb.: Professiya; 2008.

11. Penzin R.A., Shinelov Yu.G., Kireev M.V., Kustova G.L., Linkind V.A., Strelko V.V., Filipov E.A., Laskorin B.N. Development of technology for industrial synthesis of bimodal structured silica gel of the ASKM brand. Chemistry and technology of inorganic sorbents. Ashgabat: 1982.

12. Gorbunova O.V. Formation of micro- and mesoporous silica materials under conditions of sol-gel synthesis in the presence of polyethylene glycol [dissertation]. Omsk: 2014, $129 \mathrm{p}$.

13. Tumanov Yu.N. Plasma, high-frequency, microwave, and laser technologies in chemical and metallurgical processes. Moscow: FIZMATLIT, 2010.

14. Avsineeva N.K., Kalinchev V.A., Kulikov Yu.U. The use of plastics in mechanical engineering. Sat. tr. MVTU them. Bauman no. 19. Moscow: 1982. p. 6-9. (In Russian).

15. Kudryavtsev P.G., Kavalerova O.B., Pilipenko V.G., Kazakova I.L., Vorobiev O.A., Kropacheva M.V. A.S. USSR Patent No. 1787890. A method of obtaining a refractory heat-shielding material. 15.01.1993.

16. Banas R.P., Gzowski E.R., and Larsen W.T. Processing Aspects of the Space Shuttle Orbiter's Ceramic Reusable Surface Insulation. In: Ceram. Eng. and Sci. Proc. 1983;4(78): 591-610.

17. Pomogailo A.D., Rosenberg A.S., Uflyand I.E. Metal nanoparticles in polymers. Moscow: Chemistry; 2000.

18. Santos A., Ajbary M., Toldeo-Fernandez J.A., Morales-Florez V., Kherbeche A., Esquivias L. Reactivity of $\mathrm{CO}_{2}$ traps in aerogel-wollastonite composite. J. Sol-GelSci. Technol. 2008;48: 224-230.

19. Kudryavtsev P.G., Volkhin V.V. Sol-gel process and some of its technological applications, Sol-gel processes for obtaining inorganic materials. In: collection of articles. report seminar. Perm: 1991. P. 3-5. (In Russian).

20. Aegerter M.A. Leventis N. Koebel M.M. Aerogels handbook. Springer; 2011.

21. Gash A.E., Satcher J.H., Simpson R.L. Direct preparation of nanostructured energetic materials using sol-gel methods. In: Miziolek, AW, Karna Sp, Mauro JM, Vaia Ra (Eds.) Defense applications of nanomateirals. Washington DC: American Chemical Society; 2002.

22. Umbrajkar S.M., Schoenitz M., Dreizin E.L. Structural refinement in Al-MoO3 nanocomposites prepared by arrested reactive milling. Materials Research Society Symposium Proceedings. Vol. 896; Multifunctional Energetic Materials. Warrendale PA (USA): Material Research Society; 2005.

23. Son S.F., Yetter Y.A., Yang V. Introduction: Nanoscale energetic materials. Journal of Power and Propulsion and Power. 2007;23(4): 643-644.

24. Maxwell Garnett J.C. Colors in metal glasses and in metallic films. Philosophical Transactions Royal Society of London. 1904;A203: 385-420.

25. Kudryavtsev P., Figovsky O. Nanocomposite Organomineral Hybrid Materials. Scientific Israel - Technological Advantages. 2015;17(3): 7-60.

26. Carlson P. Aerogel Cherenkov counters: construction principles and applications. Nucl. Instrum. Methods Phys. Res. 1986;A248: 110-117.

27. Anderson M.L., Rolison D.R., Merzbacher C.I. Composite aerogels for sensing applications. Proc. SPIE. 1999;3790: 38-42.

28. Boday D.J. Loy D.A. Poly aniline nanofiber/silica aerogel composites with improved strength and sensor applications. Polymer Preprints. 2009;50: 282.

29. Woignier T., Reynes J., Phalippou J., Dussossoy J.L., Jacquet-Francillon N. Sintered silica aerogel: a host matrix for long live nuclear wastes. J. Non-Cryst. Solids. 1998;225: 353-357.

30. Izaak T.I., Vodyankina O.V. Macroporous monolithic materials: synthesis, properties, application. Uspekhi khimii. 2009;78(1): 80-92. 
31. Yue W., R.J.Park, A.N.Kulak, F.C.Meldrum. Macroporous inorganic solids from a biomineral template. Journal of Crystal Growth. 2006;294(1): 69-77. Available from: https://doi.org/10.1016/j.jcrysgro.2006.05.028.

32. Shabanova N.A., Popov V.V., Sarkisov P.D. Chemistry, and technology of nanodispersed oxides. Moscow: Akademkniga, 2007.

33. Tokudome Y., Fujita K., Nakanishi K., Miura K., Hirao K. Synthesis of Monolithic $\mathrm{Al}_{2} \mathrm{O}_{3}$ with Well-Defined Macropores and Mesostructured Skeletons via the Sol-Gel Process Accompanied by Phase Separation. Chem. Mater. 2007;19(14): 3393-3398. Available from: doi: 10.1021/cm063051p.

34. Rosenberg B.A. Microphase separation in hardening multicomponent polymer-oligomeric systems. Rus. chem. zhurn. 2001;45(5-6): 23-31.

35. Cahn J.W., Hilliard J.E. Free Energy of a Nonuniform System. I. Interfacial Free Energy. The Journal of Chemical Physics. 1958;28(2): 258-267. Available from: https://doi.org/10.1063/1.1744102.

36. Saito H., Kanamori K., Nakanishi K., Hirao K., Nishikawa Y., Jinnai H. Three-dimensional observation of macroporous silica gels and the study on structural formation mechanism. Colloids and Surfaces A: Physicochemical and Engineering Aspects. 2007;300(1-2): 245-252. Available from: https://doi.org/10.1016/j.colsurfa.2006.12.075.

37. Brinker C.J., Scherer G.W. In Ultrastructure Processing of Ceramics, Glass and Composites. Eds L.L. Hench, D.R. Ulrich. New York: Wiley, 1984.

38. Nakanishi K., Soga N. First Synthesis and Reactivities of Isolable Dithiiranes and Their 1-Oxides. Bull. Chem. Soc. Jpn. 1997;70(3): 509-523. Available from: https://doi.org/10.1246/bcsj.70.509.

39. Kudryavtsev P. Composition and structure of porous heat-resistant inorganic composite materials. Nanotechnologies in Construction. 2018;10(4): 75-100. Available from: doi: 10.15828/2075-8545-2018-10-4-75-100.

40. Kudryavtsev P.G. Structure of pores in solid porous bodies. Part I. Nanotechnologies in Construction. 2018;10(5): 80-103. Available from: doi: 10.15828/2075-8545-2018-10-5-80-103.

41. Kudryavtsev P.G. Structure of pores in solid porous bodies. Part II. Nanotechnologies in Construction. 2018;10(6): 80-111. Available from: doi: 10.15828/2075-8545-2018-10-6-80-111.

42. Kudryavtsev P.G. Properties of porous heat-resistant composition materials. Part I. Nanotechnologies in Construction. 2019;11(6): 623-639. Available from: doi: 10.15828/2075-8545-2019-11-6-623-639.

43. Kudryavtsev P.G. Properties of porous heat-resistant composition materials. Part II. Nanotechnologies in Construction. 2020;12(1): 15-20. Available from: doi: 10.15828/2075-8545-2020-12-1-15-20.

\section{INFORMATION ABOUT THE AUTHOR}

Pavel G. Kudryavtsev, PhD in Chemistry, Professor, Deputy Director for Research and Development KUD Industries PN Ltd Israel Nanotechnology Technology Research Center, Haifa, Israel, ORCID: https://orcid.org/0000-0002-0729-0958,

e-mail:pgkudr89@gmail.com

\section{Authors declare the absence of any competing interests.}

Received: 12.09.2020.

Revised: 05.10.2020.

Accepted: 07.10.2020. 